PROCEEDINGS OF THE

AMERICAN MATHEMATICAL SOCIETY

Volume 135, Number 2, February 2007, Pages 559-567

S 0002-9939(06)08478-4

Article electronically published on August 28, 2006

\title{
NOTES ON BRAIDZEL SURFACES FOR LINKS
}

\author{
TAKUJI NAKAMURA
}

(Communicated by Ronald A. Fintushel)

\begin{abstract}
As a generalization of pretzel surfaces, L. Rudolph has introduced a notion of braidzel surfaces in his study of the quasipositivity for pretzel surfaces. In this paper, we show that any oriented link has a braidzel surface. We also introduce a new geometric numerical invariant of links with respect to their braidzel surface and study relationships among them and other "genus" for links.
\end{abstract}

\section{INTRODUCTION}

A link is a smooth 1-manifold embedded in the 3 -sphere $S^{3}$, and a knot is a link with one connected component. It is well known that any link is the boundary of a compact, connected surface, called a spanning surface for the link. If a spanning surface for a link is orientable, then we call it a Seifert surface for the link. For $n$ integers $t_{1}, t_{2}, \ldots, t_{n}$, a spanning surface for a link is said to be a pretzel surface, denoted by $P\left(t_{1}, t_{2}, \ldots, t_{n}\right)$, if it consists of two disks joined by a $\left|t_{i}\right|$ half-twisted, unknotted vertical band $b_{i}$ for $i=1,2, \ldots, n$ as in Figure 1(a). If $t_{i}$ is positive (resp. negative), then $b_{i}$ is right-handed (resp. left-handed). Note that a pretzel surface $P\left(t_{1}, t_{2}, \ldots, t_{n}\right)$ is orientable if and only if all $t_{i}$ 's are odd or even. A link with a pretzel surface is called a pretzel link. Figure 1(b) illustrates a schematic diagram of a spine of a pretzel surface.

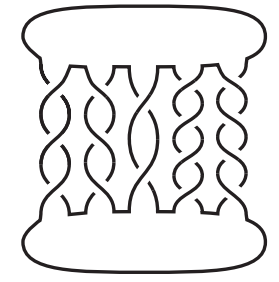

(a)

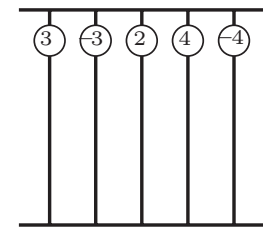

(b)

FIGURE 1.

Received by the editors May 4, 2004 and, in revised form, August 23, 2005.

2000 Mathematics Subject Classification. Primary 57M25.

Key words and phrases. Pretzel surface, braidzel surface, braidzel genus, free genus, canonical genus.

This work was supported by the 21st Century COE program "Constitution of wide-angle mathematical basis focused on knots".

(C)2006 American Mathematical Society 
We can regard the cores of the bands of a pretzel surface as the $n$-string trivial braid; see Figure 1(b). From such a point of view, L. Rudolph has introduced a notion of braidzel surfaces as a generalization of pretzel surfaces in [5] on his study of the quasipositivity for pretzel surfaces. Let $\beta$ be an $n$-string braid and $t_{1}, t_{2}, \ldots, t_{n}$ integers. A spanning surface for a link is said to be a braidzel surface, denoted by $B\left(\beta ; t_{1}, t_{2}, \ldots, t_{n}\right)$, if it consists of two disks joined by $n$ bands $b_{1}, b_{2}, \ldots, b_{n}$ such that: (1) the cores of the bands form an $n$-string braid $\beta ;(2)$ each $b_{i}(1 \leq i \leq n)$ is $\left|t_{i}\right|$ half-twisted. If $t_{i}$ is positive (resp. negative), then $b_{i}$ is righthanded (resp. left-handed). More precisely, we call it an $n$-braidzel surface. See Figure 2, which illustrates $B(\beta ; 3,-3,2,4,-4)$, where $\beta=\sigma_{1}^{2} \sigma_{3} \sigma_{4} \sigma_{3} \sigma_{2} \sigma_{1}^{-1} \sigma_{2} \sigma_{4}^{-2}$ is written by Artin's standard generators. Similar to the case of a pretzel surface, a braidzel surface $B\left(\beta ; t_{1}, t_{2}, \ldots, t_{n}\right)$ is orientable if and only if all $t_{i}$ 's are odd or even.

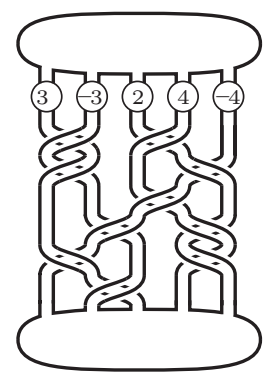

(a)

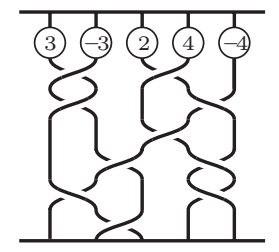

(b)

FiguRE 2.

By direct deformation, we see that any 2-braidzel surface is ambient isotopic to a twisted annulus or a twisted Möbius band. Moreover we see that any 3-braidzel surface is ambient isotopic to a pretzel surface.

We see that any 2-bridge link and any torus link has a braidzel surface by direct deformation. Moreover a twisted double of a trefoil knot and the connected-sum of a trefoil knot and its copy also have braidzel surfaces, though any pretzel knot is simple. So the question can be proposed naturally. What kinds of links have braidzel surfaces? In this paper, we give the complete answer to this question.

Theorem 1.1. Any oriented link has an oriented braidzel surface.

By considering the connected-sum of an orientable braidzel surface and the unknotted, untwisted Möbius band and some modifications, we have a corollary.

Corollary 1.2. Any unoriented link has a non-orientable braidzel surface.

This paper is organized as follows. In Section 2, we will prove Theorem 1.1. In Section 3, we will introduce a new geometric numerical invariant of links with respect to their braidzel surface, the braidzel genus. We will study relationships among them and other "genus" for links.

For the definition of standard terms in knot theory, we refer to [2] and [4]. 


\section{Proof of Theorem 1.1}

In this section, we prove our main theorem, Theorem 1.1 Hereafter we assume that all links are oriented unless otherwise stated.

A Seifert surface for a link $L$ is said to be canonical if it is obtained from a connected diagram of $L$ by applying Seifert's algorithm. Theorem 1.1 immediately follows from the next theorem.

Theorem 2.1. Any canonical Seifert surface for $L$ is ambient isotopic to a braidzel surface.

A diagram $\widetilde{L}$ is said to be flat if any Seifert circle in $\widetilde{L}$ is innermost. A canonical Seifert surface obtained from a flat diagram is called a flat Seifert surface. M. Hirasawa [1] showed that any canonical Seifert surface is ambient isotopic to a flat Seifert surface. Therefore in fact we prove the following.

Proposition 2.2. Any flat Seifert surface for $L$ is ambient isotopic to a braidzel surface.

Let $G$ be a connected, finite plane graph on the 2-sphere $S^{2}$, and $v_{0}$ a given vertex of $G$. We denote by $V(G)$ the set of vertices of $G$ and by $|V(G)|$ the number of vertices of $G$. We define the three moves for $G$ as indicated in Figure 3 as follows: The move M1 is defined as changing each multi-edge into one edge (Figure 3(a)).

Let $v\left(\neq v_{0}\right)$ be a vertex of degree two and $e, e^{\prime}$ edges incident to $v$. Assume that $e$ and $e^{\prime}$ has no common vertex except $v$. Then the move M2 is defined as changing $e, e^{\prime}$ and $v$ into one edge (Figure $3(\mathrm{~b})$ ).

Let $e_{0}$ be an edge incident to $v_{0}$ and another vertex $v$, and $e$ an edge incident to $v$ and another vertex $v^{\prime}\left(\neq v_{0}\right)$. Let $a$ be an arc on $S^{2}$ connecting $v$ and $v^{\prime}$. If the interior of a domain bounded by $v_{0} \cup e_{0} \cup v \cup e \cup v^{\prime} \cup a$ has no intersection with $G$, that is, the domain is innermost, then we replace the edge $e$ by $a$. We can regard this operation as moving the boundary of $e$ on $v$ to $v_{0}$ along $e_{0}$. The move M3 is defined as this operation (Figure 3(c)).

(a) M1:

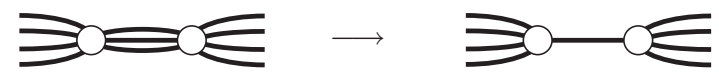

(b) M2:
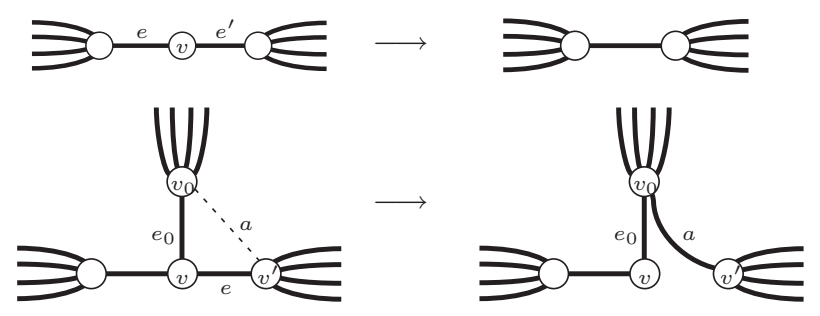

FiguRE 3.

Let $G_{0}$ be the tree of two vertices.

Lemma 2.3. Assume that $G$ has no loop and no cut-vertex. Then independent of the choice of $v_{0}, G$ can be deformed into $G_{0}$ by a finite sequence of the moves M1, M2 and M3 without deleting $v_{0}$. 
Proof. We prove Lemma 2.3 by induction on $|V(G)|$. Note that each of the moves M1, M2 and M3 does not make a loop in the resulting graph after applying them. Furthermore each of the moves M1 and M2 also does not make a cut-vertex.

Suppose $|V(G)|=2$. By applying the move M1, we may assume that $G$ has no multi-edge. Then obviously Lemma 2.3 holds. Suppose $|V(G)|=3$. Then we see that $G$ is a triangle graph after applying the move M1. By applying the move M2 to a vertex except $v_{0}$, we obtain a graph consisting of two vertices connected by two edges. Then Lemma 2.3 holds by the move M1.

Assume that Lemma 2.3 holds for any $G$ with $|V(G)|<n$ for a positive integer $n(\geq 4)$. Now suppose $|V(G)|=n$. By applying the move M1, we may assume $G$ has no multi-edge. If there exists a vertex of degree two except $v_{0}$, then by applying the move M2, we obtain a new plane graph $G^{\prime}$ with $\left|V\left(G^{\prime}\right)\right|<|V(G)|$. Thus Lemma 2.3 holds by induction assumption.

Assume that there exists no vertex of degree two in $V(G) \backslash\left\{v_{0}\right\}$. Let $v_{1}, v_{2}, \ldots, v_{k}$ be the vertices incident to $v_{0}$. Since if $k=1$, then $v_{1}$ is a cut-vertex, we may assume $2 \leq k \leq n-1$. Let $e$ be the edge incident to $v_{0}$ and $v_{i}$ for some $i(1 \leq i \leq k)$. Let $A_{i}$ be a simple arc on $S^{2}$ connecting $v_{0}$ and $v_{i}$, where $A_{i} \cap G=\left\{v_{0}, v_{i}\right\}$. Then $A_{i} \cup v_{0} \cup e \cup v_{i}$ forms a simple loop $C_{i}$ on $S^{2}$. Let $D_{i}$ and $D_{i}^{\prime}$ be the two disks of $S^{2}-\operatorname{int} N\left(C_{i}\right)$, where $N\left(C_{i}\right)$ is a tubular neighborhood of $C_{i}$ in $S^{2}$. If $D_{i} \cap G \neq \emptyset$ and $D_{i}^{\prime} \cap G \neq \emptyset$, then we call such a simple loop a cut-loop for $G$. See Figure 4. If there exists a cut-loop $C_{i}$, then applying the moves M3 with respect to $v_{i}$ makes $v_{0}$ a cut-vertex. Conversely if applying the move M3 to $G$ makes $v_{0}$ a cut-vertex, then there exists a cut-loop for $G$ on $S^{2}$.

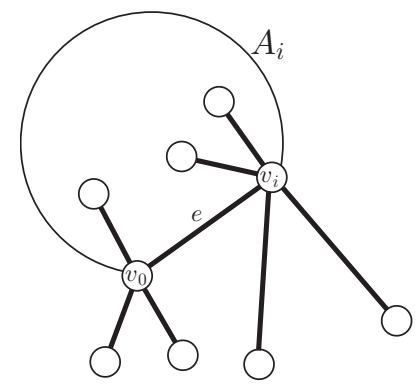

FIGURE 4.

Assume that there exist several cut-loops for $G$ on $S^{2}$. We denote the innermost one by $C_{i}$, and two disks of $S^{2}-\operatorname{int} N\left(C_{i}\right)$ by $D_{i}$ and $D_{i}^{\prime}$, again. If there exists no vertex in $D_{i}$, then $v_{0}$ and $v_{i}$ are connected by a multi-edge. If there exists no vertex incident to $v_{0}$ in $D_{i}$, then $v_{i}$ is a cut-vertex. These are contradictions. Hence there exists an integer $j \in\{1,2, \ldots, i-1, i+1, \ldots, k\}$ such that vertex $v_{j}$ is incident to $v_{0}$ in $D_{i}$. Then since $C_{i}$ is innermost, the move M3 with respect to $v_{j}$ does not make $v_{0}$ a cut-vertex.

Suppose that the degree of $v_{j}$ is $m+1$. Let $e_{0}$ be the edge connecting $v_{j}$ and $v_{0}$. For the edges incident to $v_{j}$ except $e_{0}$, we denote them by $e_{1}, e_{2}, \ldots, e_{m}$ counterclockwise around $v_{j}$ from $e_{0}$. By applying the move M3 with respect to $v_{j}$, we move the boundary of each $e_{i}$ on $v_{j}$ to $v_{0}$ along $e_{0}$ for $i=1$ to $m-1$. Then since the degree of $v_{j}$ is two in the resulting graph, we can apply the move M2 to $e_{0}, e_{m}$ and $v_{j}$. See Figure 5. Therefore we obtain a new plane graph $G^{\prime}$ with 
$\left|V\left(G^{\prime}\right)\right|<|V(G)|$. If there exists no cut-loop for $G$, then we also obtain $G^{\prime}$ with $\left|V\left(G^{\prime}\right)\right|<|V(G)|$ by applying the move M3 with respect to any vertex incident to $v_{0}$. The conclusion follows from induction assumption.

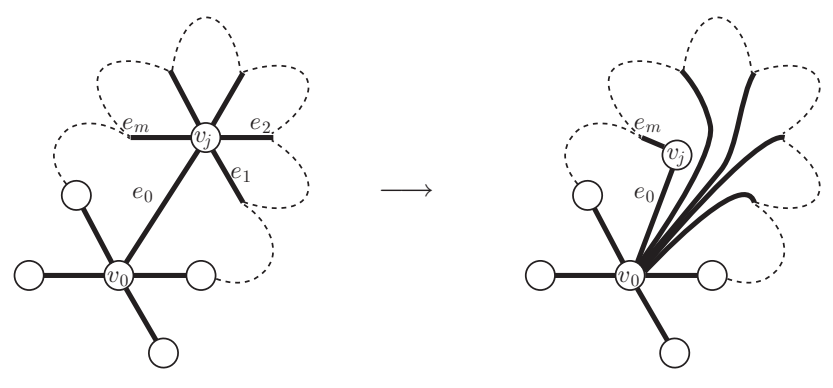

FiguRE 5 .

Translation. Before proving Proposition 2.2, we translate this argument for a plane graph into that for a spanning surface for a link. We regard each vertex as a disk and each edge as several bands whose cores form a braid as in Figure 6(a). We call such a set of bands a $\beta$-braiding-bands-set when the cores of the bands form a braid $\beta$. Let $D_{v_{0}}$ be the disk corresponding to $v_{0}$. For only the disk $D_{v_{0}}$, the cores of all bands in the braiding-bands-sets incident to $D_{v_{0}}$ form one braid $\beta$ as in Figure 6(b). Then we see that a graph $G$ in Lemma 2.3 presents a spanning surface for a link. Note that the graph $G_{0}$ presents a braidzel surface.

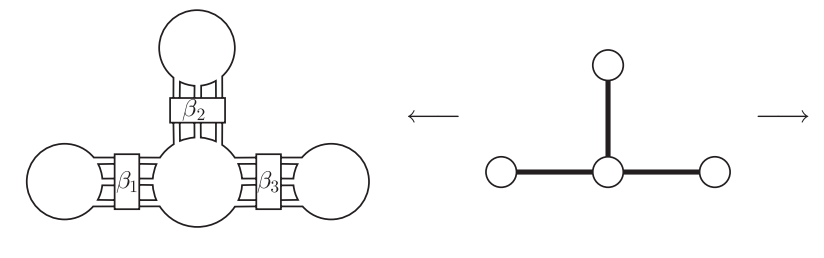

(a)

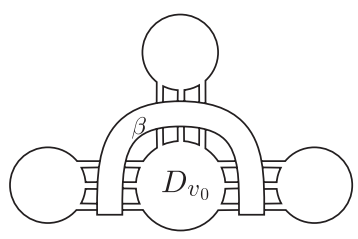

(b)

FIGURE 6.

The moves M1, M2 and M3 for a graph are corresponding to those for a spanning surface for a link depicted in Figure 7.

The move M1 is realized by the following: If there exist two disks connected by a $\beta_{1}$-braiding-bands-set and a $\beta_{2}$-braiding-bands-set, then we present them with one braiding-bands-set.

The move M2 is realized by the following deformation of a surface: Let $D$ be a disk corresponding to a vertex of degree two and $D^{\prime}$ (resp. $D^{\prime \prime}$ ) a disk connected with $D$ by a $\beta^{\prime}$-braiding-bands-set (resp. a $\beta^{\prime \prime}$-braiding-bands-set). Let $b_{1}^{\prime}, b_{2}^{\prime}, \ldots, b_{m}^{\prime}$ (resp. $b_{1}^{\prime \prime}, b_{2}^{\prime \prime}, \ldots, b_{n}^{\prime \prime}$ ) be the elements of the $\beta^{\prime}$-braiding-bands-set (resp. the $\beta^{\prime \prime}$-braiding-bands-set) according to Figure 8(a). First we thicken the band $b_{1}^{\prime}\left(\right.$ resp. $\left.b_{1}^{\prime \prime}\right)$ and thin bands $b_{2}^{\prime}, \ldots, b_{m}^{\prime}\left(\right.$ resp. $\left.b_{2}^{\prime \prime}, \ldots, b_{n}^{\prime \prime}\right)$. See Figure $8(\mathrm{~b})$. 
(a) M1:

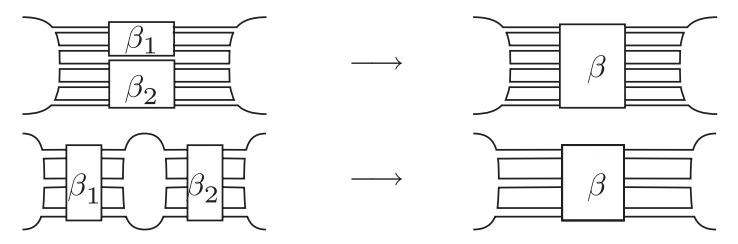

(b) M2:

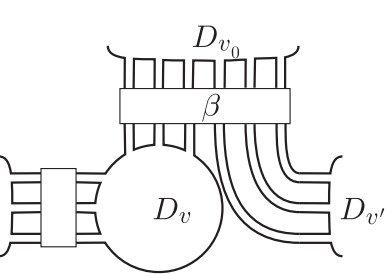

(c) M3:

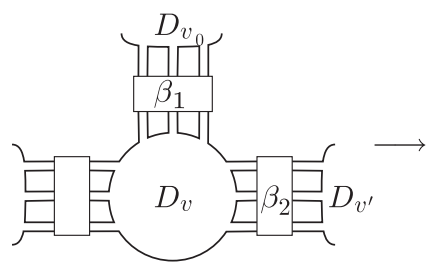

FIGURE 7.

Second, we deform $D$ as follows: Let $a_{i}^{\prime}$ (resp. $a_{i}^{\prime \prime}$ ) be a "short" subarc of $\partial D$ between $b_{i}^{\prime}$ and $b_{i+1}^{\prime}$ for $i=1,2, \ldots, m-1$ (resp. $b_{i}^{\prime \prime}$ and $b_{i+1}^{\prime \prime}$ for $i=1,2, \ldots, n-1$ ). By pushing the arcs $a_{1}^{\prime}, a_{2}^{\prime}, \ldots, a_{m-1}^{\prime}$ (resp. $\left.a_{1}^{\prime \prime}, a_{2}^{\prime \prime}, \ldots, a_{n-1}^{\prime \prime}\right)$ into the thickened band $b_{1}^{\prime \prime}$ (resp. the thickened band $b_{1}^{\prime}$ ) until they reach $\partial D^{\prime \prime}$ (resp. $\partial D^{\prime}$ ), then we see that $D^{\prime}$ and $D^{\prime \prime}$ are connected by a new braiding-bands-set, since $D$ can be regarded as being deleted.

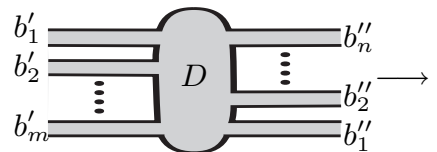

(a)

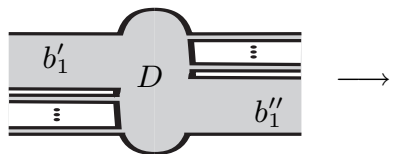

(b)

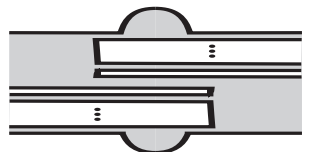

(c)

FiguRE 8.

Let $B_{1}$ be a $\beta_{1}$-braiding-bands-set incident to the disk $D_{v_{0}}$ and a disk $D_{v}$. Let $B_{2}$ be a $\beta_{2}$-braiding-bands-set incident to $D_{v}$ and another disk $D_{v^{\prime}}\left(\neq D_{v_{0}}\right)$. The move M3 is realized by sliding the bands in $B_{2}$ on $D_{v}$ to $D_{v_{0}}$ along the band in $B_{1}$ nearest to $B_{2}$ on $\partial D_{v}$. See Figure 7 (c).

Note that these moves for a spanning surface preserves the orientability. We also note that this translation completely recovers Lemma 2.3. since the edges incident to $v_{0}$ are not moved by the move M3.

Proof of Proposition 2.2. Let $\widetilde{L}$ be a reduced, connected flat diagram of a link $L$ and $S$ a flat Seifert surface obtained from $\widetilde{L}$. A flat Seifert surface $S$ consists of Seifert disks and half-twisted bands connecting them and is on $S^{2}$ except small neighborhoods of crossing points of half-twisted bands. Let $\ell$ be a simple loop on $S^{2}$ meeting $\widetilde{L}$ at two points. If any such a loop $\ell$ bounds a disk intersecting $\widetilde{L}$ in a simple arc, then we say $\widetilde{L}$ is prime. Otherwise, $\ell$ is called a separating loop. (Assume that $\ell$ meets $\widetilde{L}$ in a Seifert circle in $\widetilde{L}$.)

Assume that $\widetilde{L}$ is prime. We regard each Seifert disk and each half-twisted band of $S$ as a vertex and an edge, respectively. Then we obtain a graph $G$, which is called a Seifert graph, from $S$. A half-twisted band can be regarded as a $\beta$-braidingbands-set where $\beta$ is the 1 -string trivial braid. Then since $\widetilde{L}$ is flat and prime, we 
see that $G$ satisfies the assumption of Lemma 2.3. By Lemma 2.3 and its translation mentioned above, $G$ can be deformed into $G_{0}$. Hence we obtain a braidzel surface which is ambient isotopic to $S$.

Assume that $\widetilde{L}$ is not prime. Let $\widetilde{L}_{1}, \widetilde{L}_{2}, \ldots, \widetilde{L}_{n}$ be $n$ subdiagrams of $\widetilde{L}$ as prime factors and $S_{1}, S_{2}, \ldots, S_{n}$ subsurfaces of $S$ obtained from $\widetilde{L}_{1}, \widetilde{L}_{2}, \ldots, \widetilde{L}_{n}$, respectively. Then there exist $n-1$ separating loops on $S^{2}$. By deforming $\widetilde{L}$ with $S$, we may assume that the separating loops are concentric. Note that this deformation preserves the flatness of $\widetilde{L}$, hence of $S$. We denote by $\ell_{i}$ the $i$-th separating loop from the innermost one; see Figure 9(a). (Each rectangle in Figure 9(a) contains $S_{i}$.) Let $D_{i}$ be a Seifert disk of $S$ such that $\ell_{i} \cap S \subset D_{i}(1 \leq i \leq n-1)$. Let $D_{n}$ be a Seifert disk in $S_{n}$ as in Figure 9(a). To be precise, we consider $S$ as $S_{1} \sharp_{D_{1}} S_{2} \sharp_{D_{2}} \cdots \sharp_{D_{n-1}} S_{n}$, where $S_{i} \sharp_{D_{i}} S_{i+1}$ means the connected sum of $S_{i}$ and $S_{i+1}$ along $D_{i}$. We deform $S$ into a braidzel surface as follows: First, we designate $D_{1}$ as the disk corresponding to the vertex $v_{0}$ in Lemma 2.3. Second, we deform $S_{1}$ into a braidzel surface $B_{1}$ as in the translation of the proof of Lemma 2.3. Then we see that it can be done in the disk bounded by $\ell_{1}$ on $S^{2}$ which contains $S_{1}$. Therefore we can regard $S$ as $B_{1} \sharp_{D_{1}} S_{2} \sharp_{D_{2}} \cdots \sharp_{D_{n-1}} S_{n}$; see Figure 9(b). Next we slide $B_{1}$ so that it is connected with $S_{n}$ along $D_{n}$, and denote by $D_{1}^{\prime}$ the other disk of $B_{1}$. Then we have $S=S_{2} \sharp_{D_{2}} \cdots \sharp_{D_{n-1}} S_{n} \sharp_{D_{n}} B_{1}$; see Figure 9(c). Similarly, we deform $S_{2}$ into a braidzel surface $B_{2}$ in the disk bounded by $\ell_{2}$ on $S^{2}$ which contains $S_{2}$. We also slide $B_{2}$ so that it is connected with $B_{1}$ along $D_{1}^{\prime}$ and denote by $D_{2}^{\prime}$ the other disk of $B_{2}$. Then we obtain $S=S_{3} \sharp_{D_{3}} \cdots \sharp_{D_{n-1}} S_{n} \sharp_{D_{n}} B_{1} \sharp_{D_{1}^{\prime}} B_{2}$; see Figure 9(d). By proceeding with this procedure, we have $S=B_{1} \sharp_{D_{1}^{\prime}} B_{2} \sharp_{D_{2}^{\prime}} \cdots \sharp_{D_{n-1}^{\prime}} B_{n}$. Finally, we obtain a braidzel surface by applying $n-2$ times of the deformation in the translation of the move M2. The proof is completed.

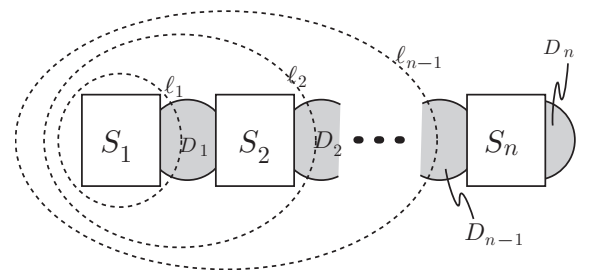

(a)

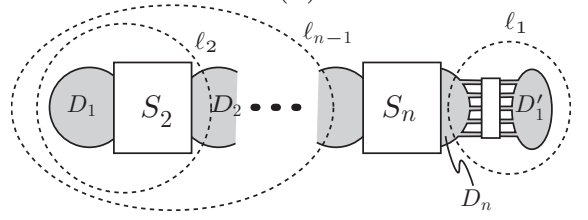

(c)

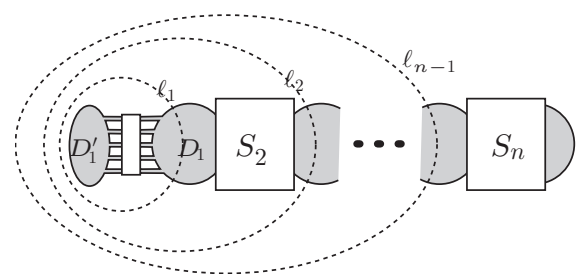

(b)

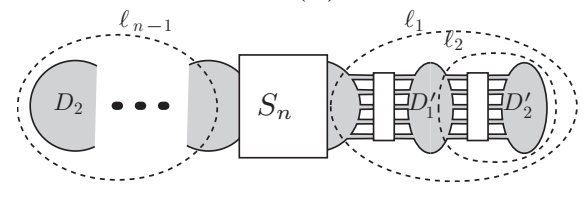

(d)

FiguRE 9.

\section{BRAIDZEL GENUS FOR LINKS}

By our main theorem, we can define a new geometric numerical invariant for links with respect to their braidzel surfaces. 
Definition 3.1. The minimal genus among all oriented braidzel surfaces for a link $L$ is defined to be the braidzel genus for $L$, denoted by $g_{b}(L)$.

Similarly, the minimal genus among all canonical Seifert surfaces for $L$ is called the canonical genus for $L$, denoted by $g_{c}(L)$. A Seifert surface $S$ of a link $L$ is said to be free if the fundamental group of the complement of $S$ is a free group. This condition is equivalent to the fact that $S^{3}-\operatorname{int} N(S)$ is a handlebody. The minimal genus among all free Seifert surfaces for $L$ is called the free genus for $L$, denoted by $g_{f}(L)$. We see that any braidzel surface is free. By Theorem 2.1, we have $g(L) \leq g_{f}(L) \leq g_{b}(L) \leq g_{c}(L)$, where $g(L)$ is the minimal genus for $L$ in the usual sense. Obviously, the equality holds for the links with canonical Seifert surfaces giving $g(L)$. However we obtain the following.

Theorem 3.2. There exist infinitely many simple fibered knots with braidzel genus less than their canonical genus, $g_{b}(K)<g_{c}(K)$.

Proof. Let $K_{m}$ be a knot as in Figure 10(a). (The rectangle in Figure 10(a) illustrates $|m|$ full-twists.) The author showed in [3] that $K_{m}$ is a simple fibered knot with $g_{f}\left(K_{m}\right)=3$ and $g_{c}\left(K_{m}\right) \geq 4$. He also showed that $K_{m}$ is not equivalent to $K_{m^{\prime}}$ if $m \neq m^{\prime}$. It is known that a fiber surface for a fibered knot is unique up to ambient isotopy, is a minimal genus Seifert surface and is free. Now we obtain a braidzel surface of genus three for $K_{m}$ as in Figure 10(b), hence $g_{b}\left(K_{m}\right)=3$. This completes the proof.

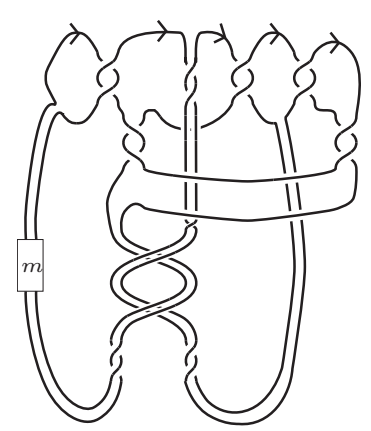

(a)

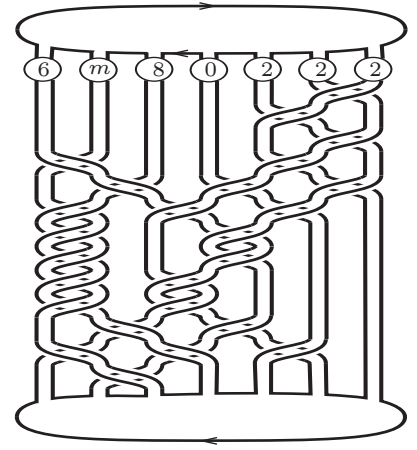

(b)

FiguRe 10.

We also obtain the following.

Theorem 3.3. There exist infinitely many links with braidzel genus greater than their free genus, $g_{f}(L)<g_{b}(L)$.

Proof. Let $L_{m}$ be a 3-component link as in Figure 11(a), where the rectangle in the figure illustrates $|m|$ full-twists. Then $L_{m}$ has a Seifert surface $S_{m}$ with the first Betti number two, as in the lower part of Figure 11(a). It consists of the knotted annulus whose core is a trefoil knot and an $|m|$ full-twisted band. We see that $S_{m}$ is free, since a tubular neighborhood of $S_{m}$ can be regarded as a tubular neighborhood of a trefoil knot with an unknotting tunnel. Hence we have 
$g_{f}\left(L_{m}\right)=\left(1-\mu\left(L_{m}\right)+B_{1}\left(S_{m}\right)\right) / 2=0$, where $\mu\left(L_{m}\right)$ is the number of components of $L$ and $B_{1}\left(S_{m}\right)$ is the first Betti number of $S_{m}$. It is known that any oriented braidzel surface of the first Betti number two is a 3-braidzel surface, hence a 3strand pretzel surface. The bridge index of a 3 -strand pretzel link is at most three. The bridge index of $L_{m}$ is, however, at least four, since $\mu\left(L_{m}\right)=3$ and one of the components of $L_{m}$ is a trefoil knot. Thus the first Betti number of any oriented braidzel surface for $L_{m}$ is greater that two, hence $g_{b}\left(L_{m}\right) \geq 1$. Note that we see $g_{b}\left(L_{m}\right)=1$, since $L_{m}$ has the braidzel surface as in Figure 11(b), where the rectangle means $|m|$ full-twists of two bands.

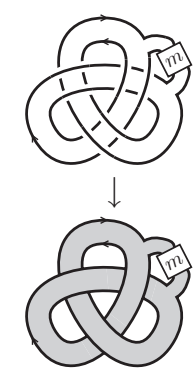

(a)

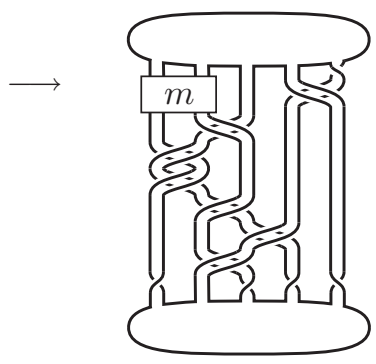

(b)

Figure 11.

\section{REFERENCES}

[1] M. Hirasawa, The flat genus of links, Kobe J. Math., 127 (1995), 155-159. MR.1391192 (97g:57006)

[2] A. Kawauchi, A survey of knot theory, Birkhäuser-Verlag, Basel, 1996. MR.1417494 (97k:57011)

[3] T. Nakamura, On canonical genus of fibered knot, J. Knot Theory Ramifications, 11 (2002), 341-352. MR 1905689 (2003b:57009)

[4] D. Rolfsen, Knots and links, Mathematics Lecture Series, No. 7, Publish or Perish, Inc., Berkeley, Calif., 1976. MR0515288 (58:24236)

[5] L. Rudolph, Quasipositive pretzels, Topology Appl. 115 (2001), no.1, 115-123. MR.1840734 (2003a:57016)

Osaka City University Advanced Mathematical Institute, Sugimoto 3-3-138, SumiyoSHI-KU, OSAKA 558-8585, JAPAN

Current address: Research Center for Physics and Mathematics, Faculty of Engineering I, Osaka Electro-Communication University, Hatsucho18-8, Neyagawa, Osaka 572-8530, Japan

E-mail address: n-takuji@isc.osakac.ac.jp 